contributes to this susceptibility. This study identifies these microRNAs as potential therapeutic targets in ICUAP.

\section{S117 RSIV. F/HN-MEDIATED GENE THERAPY ENABLES LUNGS TO PRODUCE THERAPEUTICALLY RELEVANT LEVELS OF FVIII}

${ }^{1} \mathrm{KM}$ Pytel, ${ }^{1} \mathrm{MC}$ Paul-Smith, ${ }^{2} \mathrm{~J}$ Mclntosh, ${ }^{1} \mathrm{M}$ Chan, ${ }^{1} \mathrm{C}$ Meng, ${ }^{3} \mid$ Pringle, ${ }^{3} \mathrm{~L}$ Davis, ${ }^{4} \mathrm{M}$ Inoue, ${ }^{4} \mathrm{M}$ Hasegawa, ${ }^{3} \mathrm{SC}$ Hyde, ${ }^{3} \mathrm{DR}$ Gill, ${ }^{2} \mathrm{AC}$ Nathwani, ${ }^{1} \mathrm{EWFW}$ Alton, ${ }^{1} \mathrm{U}$ Griesenbach. ${ }^{1}$ National Heart and Lung Institute, Imperial College London, London, UK; ${ }^{2}$ University College London Cancer Institute, London, UK; ${ }^{3}$ Gene Medicine Research Group, University of Oxford, Oxford, UK: ${ }^{4} D N A V E C$, Japan

\subsection{6/thoraxjnl-2015-207770.123}

We have previously shown that lung when treated with Sendai virus-mediated gene transfer can produce secreted proteins and release them into the circulation (Griesenbach et al., Mol Therapy 2002). Despite the high levels of transduction efficiency the gene expression is transient and repeated administration is not feasible due to induction of immune responses. To overcome these barriers we developed a lentiviral vector specifically pseudotyped with the Sendai virus envelope proteins $\mathrm{F}$ and $\mathrm{HN}$ (rSIV. F/HN) to allow efficient transduction of the airways. Stable expression for $>20$ months after a single dose and efficient transduction after repeated administration despite detection of anti-rSIV. F/HN neutralising antibodies make the vector an attractive candidate for a large range of disease indications. Here, we first transduced mouse lung with rSIV. F/HN carrying the secreted reporter gene Gaussia luciferase (GLux) or a control virus by nasal instillation (1e6 transduction units (TU)/mouse, $n$ $=5-6$ /group). Persistent levels of GLux expression were detectable in lung (3 logs above control) and broncho-alveolar lavage fluid (BALF, 4 logs above control) for at least 12 months. Importantly, even this modest dose of virus lead to significant $(\mathrm{p}<$ $0.01)$ levels of GLux in serum (274 \pm 72 RLU/ul, control: $41 \pm$ $6 \mathrm{RLU} / \mathrm{ul}$ ) which persisted for at least 12 months further supporting the hypothesis that the lung is a suitable, non-invasive factory for production of secreted proteins. Gene therapy strategies for haemophilia have focussed on intravenous or intramuscular delivery of the gene transfer agent. Here, we treated the murine lung with rSIV. F/HN carrying the FVIII cDNA (1.6e8$3.4 \mathrm{e} 8 \mathrm{TU} / \mathrm{mouse}$,) or placebo and assessed whether therapeutically relevant levels of FVIII can be produced. Significant $(\mathrm{p}<$ 0.05 ) and dose-related levels of FVIII were detectable in lungs and BALF 10 and 28 days post-transduction. Dose-related levels of FVIII were also detectable in plasma, which reached a therapeutically relevant level of $3 \%$ of normal 1 month after gene transfer. These data support the concept that rSIV. F/HN-mediated transduction of lungs can produce therapeutically relevant and persistent levels of recombinant protein in blood.

\section{REFERENCE}

1 Griesenbach U, Cassady RL, Ferrari $S$ et al. The nasal epithelium as a factory for systemic protein delivery. Mol Ther. 2002;5:98-103

\section{S118 CIRCADIAN GLUCOSE PATTERNS IN ADULT CARDIOTHORACIC TRANSPLANT RECIPIENTS}

${ }^{1} \mathrm{~A}$ Nixon, ${ }^{1} \mathrm{~S}$ Manduell, ${ }^{2} \mathrm{~B}$ Issa, ${ }^{1} \mathrm{M}$ Al-Aloul. ${ }^{1}$ Cardiopulmonary Transplant Unit, Wythenshawe Hospital, Manchester, UK; ${ }^{2}$ Department of Diabetes and Endocrinology, Wythenshawe Hospital, Manchester, UK

10.1136/thoraxjnl-2015-207770.124
Introduction New onset diabetes after transplantation (NODAT) is a well-known complication of immunosuppressive therapy and is associated with excess morbidity and mortality. Early identification and treatment of impaired glucose regulation (IGR) is crucial to help prevent or delay the development of NODAT and its associated complications.

Aim To define circadian glucose patterns of cardiothoracic transplant (CTTx) recipients using a continuous glucose monitoring system (CGMS) and compare the findings with conventional tests for diabetes.

Method Cross-sectional study in a CTTx outpatient clinic. CGMS was used to construct circadian glucose profiles. Those with CGMS values in excess of $7.7 \mathrm{mmol} / \mathrm{l}$ were asked to complete oral glucose tolerance tests (OGTT) and HbA1c.

Participants Convenience sampling was used to identify 12 stable CTTx recipients ( 2 heart, 3 single lung and 7 double lung Tx; 9 male; mean [SD] age 58 [8] years, BMI 28.6 [4.9] $\mathrm{kg} / \mathrm{m}^{2}$, daily prednisolone dose 11.5 [2.2] mg; 4 on tacrolimus vs 8 on cyclosporine; median 477 days since $\mathrm{Tx}$ ).

Results None had symptoms of hyperglyaemia. CGMS duration range: 37 to $183 \mathrm{hrs} /$ patient. A significant difference was seen between mean morning (06.00-12.00 hrs) and evening (14.00-20.00 hrs) glucose values (5.8 [1.2] vs 7.6 [1.4] mmol/l; $\mathrm{p}<0.001$, Figure 1). On CGMS data all participants had glucose values $>7.7 \mathrm{mmol} / \mathrm{l}$. Three $(25 \%)$ had glucose values $>11.1$ and $<3.5 \mathrm{mmol} / \mathrm{l}$ on CGMS and were diagnosed with impaired glucose tolerance on OGTT. Compared with 9 normal OGTT patients, the IGT group displayed a higher number of hyperglycaemic episodes/day and a greater $\%$ of time above 7.8 $\mathrm{mmol} / \mathrm{l}$. No cases of impaired fasting glycaemia or NODAT were identified using OGTT or HbA1c.

\section{Circadian Glucose of Participants According to OGTT status}

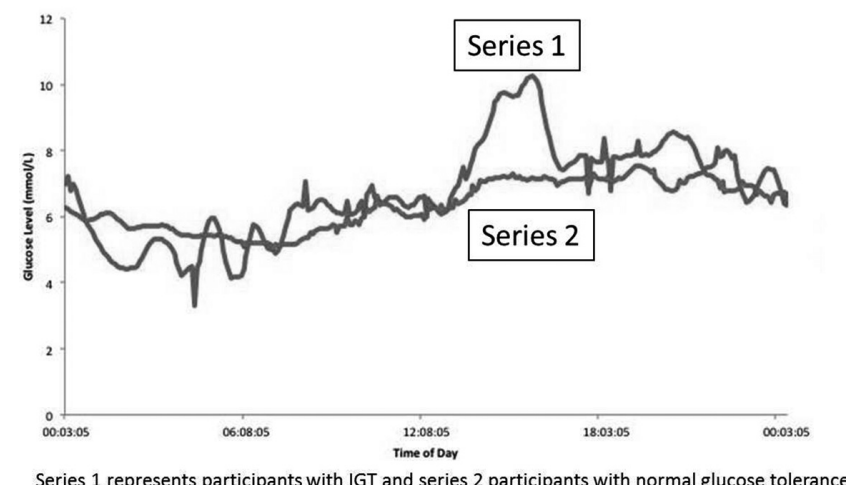

Abstract S118 Figure 1

Conclusion Findings of this pilot study emphasise the importance of improving screening for IGR in CTTx recipients. We identified diurnal variation in glucose patterns, with higher glucose values in the afternoon and evening than morning, which has implications for timing of random glucose sampling in clinic. Poor correlation was found between CGMS and conventional diagnostic tests for diabetes which may not be sensitive enough to identify IGR in CTTx recipients. This merits further investigation in a larger cohort. 